\title{
Efficiency of Spinal Anesthesia versus General Anesthesia for Minimal Invasive Single Level Transforaminal Lumbar Interbody Fusion: A Retrospective Analysis of 178 Patients
}

\author{
Ameya Rangnekar, Goparaju VNR Praveen, Amit Chugh, Saijyot Raut, Vishal Kundnani \\ Department of Orthopedics, Bombay Hospital \& Medical Research Center, Mumbai, India
}

Received: September 11, 2021

Revised: October 17, 2021

Accepted: November 29, 2021

Corresponding Author:

Ameya Rangnekar, DNB

Department of Orthopedics,

Bombay Hospital \& Medical

Research Center, 12 Vitthaldas

Thackersey Marg, New Marine

Lines, Mumbai, Maharashtra

400020, India

Tel: $+91-9098457233$

Fax : +91-2222079485

E-mail:dr.r.ameya@gmail.com
Objective: To evaluate the efficacy of spinal anesthesia in patients undergoing minimal invasive single level transforaminal lumbar interbody fusion (MIS TLIF) surgery and to compare the results with that of general anesthesia.

Method: 178 patients were included in the study, 86 were in general anesthesia and 92 were in spinal anesthesia. Patients aged between 20 to 70 years who had undergone MIS TLIF not responding to 6 weeks of conservative treatment were included. The routine steps of anesthesia for both general and spinal anesthesia were adhered. The visual analogue scale, blood loss, duration of surgery, time from entering operation theatre to time of incision, time of bandaging to exit from operation theatre, time of stay in Post Anesthesia Care Unit (PACU), nausea/vomiting, urinary retention, duration of stay in hospital, peri-operative complications were compiled and assessed. Appropriate statistical analysis was applied.

Results: The mean time for entering the operation theatre to the incision; mean time from bandaging to the exit; mean PACU time and the mean hospital stay were significantly lower in the spinal anesthesia group $(p<0.05)$. The other parameters are comparable except, urinary retention which was significantly higher in spinal anesthesia group $(p<0.05)$.

Conclusion: Spinal anesthesia offers efficient operating room functioning with decreasing overall operation theatre time. It is very efficient alternative technique to general anesthesia which can be considered for elective lumbar surgeries with a lower late of adverse events especially at lower lumbar levels.

Key Words: Spinal anesthesia, General anesthesia, Lumbar surgery, Visual analog scale, Minimal invasive single level transforaminal lumbar interbody fusion

\section{INTRODUCTION}

Unstable spine is most common indication for lumbar fusion surgeries. With advent of minimal invasive techniques, minimal invasive lumbar interbody fusion is in vogue. Various anesthetic techniques are used for performing lumbar spinal surgeries. They are performed either in general or regional anesthesia. Endotracheal general anesthesia is the most commonly used technique [1]. The main advantages of general anesthesia being that longer duration surgeries can be performed with secured airway in the prone position $[2,3]$.

The advantages in favour of spinal anesthesia being rapid

Copyright @ 2022 Korean Minimally Invasive Spine Surgery Society

This is an Open Access article distributed under the terms of the Creative Commons Attribution Non-Commercial License (http://creativecommons.org/licenses/by-nc/4.0/) which permits unrestricted non-commercial use, distribution, and reproduction in any medium, provided the original work is properly cited. 
onset, lesser blood loss, lesser thrombotic events, pulmonary complications and other neurological cognitive dysfunctions. Other benefits being that the patient can spontaneously breathe and can reposition themselves avoiding compression injuries during the procedure [4-6].

Few studies have been done which compare general anesthesia and spinal anesthesia in lumbar spinal surgeries and have reported much shorter surgical time, postoperative pain, less time in recovery room, lesser incidence of urinary retention, postoperative nausea vomiting and most importantly less financial implications in spinal anesthesia $[7,8]$.

Despite encouraging results in favour of spinal anesthesia, spinal anesthesia does not come without risk, and there is (at least to date) no clear evidence to delineate the difference in morbidity and mortality between the two approaches [9]. Hence in our study we aimed to study spinal anesthesia as an alternate option to do lumbar fusion surgeries.

\section{MATERIALS AND METHODS}

The present comparative study was a retrospective study conducted at the Department of Orthopedics, Bombay Hospital \& Research Centre, Mumbai during the study period from April 2018 to April 2020. We included 178 patients who underwent single-level primary minimal invasive transforaminal lumbar interbody fusion (MIS TLIF) for degenerative lumbar spine disorders (degenerative lumbar canal stenosis with instability, prolapsed intervertebral disc, degenerative/dysplastic/isthmic spondylolisthesis) during the study period.

Patient with age between 20 and 70 years of either gender, undergoing minimal invasive single level lumbar interbody fusion at lower lumbar levels (L3-L4, L4-L5, L5-S1), not responding to 6 weeks of conservative therapy and having mechanical low back pain with radiculopathy with claudication with/ without neurodeficit with willingness to provide their verbal consent for allowing to use their data for research purpose were included in the study.

Patient who had undergone any revision spine surgery, having tumour, infection or other pathological causes, extraspinal cause of back pain/radiculopathy, patients who required multi-level surgery, patient requiring surgery at L1-L2 or L2L3 levels and those not providing their willingness to provide consent for participation in the study were excluded. All surgeries were performed by a single surgeon in a single institute, managed by a single anesthesiologist with similar surgical and anesthetic techniques. Demographic characteristics and American Society of Anesthesiologists (ASA) physical status of the patients were all recorded.

According to the inclusion and exclusion criteria, after counselling for surgery the patients who fit the deemed criteria for study were offered both choices to choose either spinal or general anesthesia. They were thoroughly counselled and explained pros and cons associated with each technique and allowed to opt anesthesia as per their choice. Since the anesthesia was as per patient's demand, the comorbidities were not considered for evaluation. The choice of anesthesia was chosen by the patient and not observer of the study. 178 patients satisfied the selection criteria. These patients were further divided into two groups based on the anesthesia given. Of these, 92 patients underwent MIS TLIF under spinal anesthesia and 86 under general anesthesia.

\section{Anesthesia Technique}

Patients who received general anesthesia were given a combination of nitrous oxide, desflurane, propofol, sevoflurane, halothane and isoflurane. After intubating, they were positioned prone. Post completion of general anesthesia and discontinuation of anesthetic drugs, $100 \%$ oxygen was administered. Patients were then transferred to PACU after appropriate extubation. PACU nursing staff monitored patients until they were alert, responsive and stable before their transfer to ward. Intravenous analgesics was administered to the patients during their stay in PACU.

Spinal anesthesia administered patients were first given a 500 milliliters infusion of ringer lactate solution 10-15 minutes before giving spinal anesthesia. After entering operating room patient was put in seated position. Local infiltration of $3 \mathrm{~mL}$ of $2 \%$ lidocaine was given, SA was achieved via lumbar puncture, using a needle size of 25 gauze most commonly. After visualization of spinal fluid, bupivacaine was injected in combination with fentanyl into the intrathecal space. Bupivacaine was given as $15 \mathrm{mg}$ dose of a $0.75 \%$ bupivacaine in $8.25 \%$ dextrose solution. $25 \mu \mathrm{g}$ of fentanyl was given in combination with bupivacaine, in order to increase the of the spinal anesthesia antinociceptive effect. Once the spinal anesthesia had been given, adequate anesthesia was verified on the lower back and extremities after the patient was put into a supine position. The patient was then turned into the prone position on the operating table. Oxygen was administered via nasal cannula and propofol infusion assured sedation. Propofol was discontinued at completion of the procedure and the patient was transferred to the PACU for recovery. The patients remained in the PACU till hemodynamic stability was confirmed, followed by transfer 
to the ward.

Visual analogue score for pain, duration of surgery, blood loss, time from entering the operation theatre to incision, time from bandaging to exit, PACU time, duration of hospital stay, urinary retention and adverse events were our outcome measures.

A customized proforma designed for the purpose of the study was used for collecting the data of the patients. No personal details of the patients were disclosed. Only data needed for the purpose of the research paper was included. The present study was not funded by any company or institution and also no additional tests/procedures, etc. were conducted for the specific requirement of the study, so there was no financial burden on the patient and/or on the institution. Researcher bore all the expenses towards the conduct of the study.

The mean comparison between the two groups was done using independent samples test and comparison of proportions was done using Pearson Chi-square test. A p-value of $<0.05$ was taken as statistically significant.

\section{RESULTS}

178 patients were included in the study undergoing MIS TLIF under general or spinal anesthesia during the study period. Of these patients, 86 patients underwent surgery under general anesthesia and 92 under spinal anesthesia.

In both the groups, majority of the patients were in the age group more than 40 years, $95.3 \%$ in general anesthesia group and $93.5 \%$ in the spinal anesthesia group. The mean age of patients in the general anesthesia group was $58.36 \pm 9.12$ years and in the spinal anesthesia group was $56.90 \pm 10.39$ years. The difference was found to be statistically not significant ( $\mathrm{p}=0.323)$, showing a comparable mean between the two groups (Table 1).

There was a female predominance in both the groups (54.7\% in general anesthesia vs. $60.9 \%$ in spinal anesthesia group). The distribution of male and female was comparable between the two groups ( $\mathrm{p}=0.401)$ (Table 1$)$.

The indication for surgery in our study were degenerative, isthmic, lumbar canal stenosis with instability and prolapse of intervertebral disc in both the groups. And majority of the patients underwent surgery due to degeneration ( $45.3 \%$ in general anesthesia vs. $48.9 \%$ in spinal anesthesia) (Table 2).

The mean duration of surgery (general anesthesia 148.95 \pm 17.15 minutes vs. spinal anesthesia $147.55 \pm 17.29$ minutes) and mean blood loss (general anesthesia $111.22 \pm 111.74 \mathrm{~mL}$ vs. spinal anesthesia $108.69 \pm 108.45 \mathrm{~mL}$ ) were comparable between the two groups ( $p>0.05$ ). The extent of spinal anesthesia was obtained

Table 1. Patient demographic profile

\begin{tabular}{lccc}
\hline Parameter & $\begin{array}{c}\text { General anesthesia } \\
(\mathrm{n}=86)\end{array}$ & $\begin{array}{c}\text { Spinal anesthesia } \\
(\mathrm{n}=92)\end{array}$ & p-value \\
\hline Age $( \pm$ SD) years & $58.36 \pm 9.12$ & $56.90 \pm 10.39$ & $0.323, \mathrm{NS}$ \\
Gender & & & \\
$\quad$ Female & $47(54.7 \%)$ & $56(60.9 \%)$ & \\
$\quad$ Male & $39(45.3 \%)$ & $36(39.1 \%)$ & \\
\hline
\end{tabular}

NS: not significant.

Table 2. Clinical parameters

\begin{tabular}{|c|c|c|c|}
\hline Parameter & General anesthesia $(n=86)$ & Spinal anesthesia $(n=92)$ & $p$-value \\
\hline \multicolumn{4}{|l|}{ Level of surgery } \\
\hline L3-L4 & $9(10.5 \%)$ & $7(7.6 \%)$ & \\
\hline L4-L5 & $50(58.1 \%)$ & $59(64.1 \%)$ & \\
\hline L5-S1 & $27(31.4 \%)$ & $26(28.3 \%)$ & \\
\hline \multicolumn{4}{|l|}{ Indication for surgery } \\
\hline Degenerative & $39(45.3 \%)$ & $45(48.9 \%)$ & \\
\hline Isthmic & $16(18.6 \%)$ & 17 (18.5\%) & \\
\hline LCS with instability & $20(23.3 \%)$ & $22(23.9 \%)$ & \\
\hline Prolapse of intervertebral disk & $11(12.8 \%)$ & $8(8.7 \%)$ & \\
\hline Duration of surgery $( \pm S D) \min$ & $148.95 \pm 17.15$ & $147.55 \pm 12.29$ & $0.589, \mathrm{NS}$ \\
\hline Blood loss $( \pm S D) \mathrm{mL}$ & $111.22 \pm 111.74$ & $108.69 \pm 108.45$ & $0.879, \mathrm{NS}$ \\
\hline Time of entering OT to incision ( $\mathrm{min}$ ) & $41.80 \pm 32.39$ & $27.55 \pm 5.27$ & $0.001^{*}$ \\
\hline Time from bandaging to exit & $16.98 \pm 4.96$ & $6.85 \pm 3.03$ & $0.001^{*}$ \\
\hline Post Anaesthesia Care Unit min & $57.14 \pm 19.35$ & $36.79 \pm 7.32$ & $0.001^{*}$ \\
\hline Hospital stay (days) & $3.05 \pm 0.67$ & $1.61 \pm 0.55$ & $0.001^{*}$ \\
\hline
\end{tabular}

NS: not significant.

${ }^{*} p<0.05$ 
up to D10 in majority of the cases (Table 2).

The mean time from entering the operation theatre to the incision was significantly shorter in spinal anesthesia group (41.80 \pm 32.39 minutes general anesthesia vs. $27.55 \pm 5.27 \mathrm{~min}-$ utes spinal anesthesia, $\mathrm{p}<0.05$ ). Similarly, the mean time from bandaging to the exit was also significantly shorter in the spinal anesthesia group (16.98 \pm 4.96 minutes general anesthesia vs. $6.85 \pm 3.03$ minutes spinal anesthesia, $\mathrm{p}<0.05$ ) (Table 2).

The mean PACU time in the general anesthesia group was $57.14 \pm 19.35$ minutes and in the spinal anesthesia group it was $36.79 \pm 7.32$ minutes, which was significantly lower in the spinal anesthesia group $(\mathrm{p}<0.05)$ (Table 2$)$.

The mean hospital stay was significantly shorter in the spinal anesthesia group ( $1.61 \pm 0.55$ days vs. $3.05 \pm 0.67$ days in general anesthesia), $\mathrm{p}<0.05)$ (Table 2).

The mean preoperative $(\mathrm{p}=0.251)$ and postoperative visual analogue scale (VAS) was comparable between the two groups at immediate post op ( $\mathrm{p}=0.071), 3$ months ( $\mathrm{p}=0.068)$, and 12 months ( $\mathrm{p}=0.064)$ follow-ups ( $\mathrm{p}>0.05$ ) (Table 3 ).

The incidence of nausea/vomiting was comparable between the two groups ( $\mathrm{p}=0.113$ ), but the incidence of urinary retention was significantly higher in the spinal anesthesia group in comparison to the general anesthesia group (20.7\% spinal anesthesia vs. 5.8\% general anesthesia) $(\mathrm{p}<0.05)$. The other complications encountered in our study were screw malposition, dural tear, screw loosening, cage slippage and implant failure seen in very less number of patients in both the groups and were comparable in both spinal as well as general anaethesia patients (Table 4).

\section{DISCUSSION}

Despite all the comparative studies on general and spinal anesthesia, there is still controversy on the influence of these two different anesthesia methods on perioperative outcome of surgery as there are not many studies done to compare spinal anesthesia advantages over general anesthesia. So considering this lacunae in the literature, we undertook the present study at

Table 3. Visual analogue scale score

\begin{tabular}{lccc}
\hline & \multicolumn{3}{c}{ Visual analogue scale score } \\
\cline { 2 - 4 } & SA & GA & p-value \\
\hline Immediate pre op & 3.94 & 4.15 & 0.251 \\
Immediate post op & 1.68 & 1.82 & 0.071 \\
3 months & 1.64 & 1.74 & 0.068 \\
12 months & 0.52 & 0.54 & 0.064 \\
\hline
\end{tabular}

SA: spinal anesthesia, GA: general anesthesia. our institution to evaluate the efficacy of spinal anesthesia and general anesthesia in terms of clinical and surgical outcome in patients undergoing single level minimal invasive lumbar interbody fusion surgery.

In the present study with a mean age was comparable between the two groups $(\mathrm{p}>0.05)$. There was a comparable distribution of males and females in our study with a female predominance in each of the two groups. Studies done by Jellish et al. [10], McLain et al. [11], Dashtbani et al. [12], and Sadrolsadat et al. [13] also found comparable demographic variables in their studies. While in the study done by Papadopoulos et al. [14] a slightly higher age was seen in patients undergoing general anesthesia, with a male preponderance in both the groups.

The mean preoperative $(\mathrm{p}=0.251)$ and postoperative VAS was comparable between the two groups at immediate post op ( $\mathrm{p}=0.071), 3$ months $(\mathrm{p}=0.068)$ and 12 months $(\mathrm{p}=0.064)$ follow-ups ( $p>0.05$ ). While in Dashtbani et al. [12] study the mean preoperative VAS was significantly higher in general anesthesia group.

Mean blood loss reported in our study was also comparable, which is supported by the study done by Dashtbani et al. [12] While Sadrolsadat et al. [13] and Jellish et al. [10] found a significantly higher blood loss in general anesthesia group, which is contradictory to our findings.

The duration of surgery in the present study was comparable between the two groups, Sadrolsadat et al. [13] and Dashtbani et al. [12] and also found a comparable mean duration of surgery in their study, while studies done by Jellish et al. [10] and McLain et al. [11] reported a significantly longer duration of surgery in general anesthesia group $(\mathrm{p}<0.05)$.

However in our study, there was shorter total anesthesia time in the spinal anesthesia group. Though the duration of surgery is a large component of this parameter, results remained significant when adjustment was made for operative time. This is due to the reason as we recorded two additional time points,

Table 4. Complications

\begin{tabular}{lccc}
\hline Parameter & $\begin{array}{c}\text { General anesthesia } \\
(\mathrm{n}=86)\end{array}$ & $\begin{array}{c}\text { Spinal anesthesia } \\
(\mathrm{n}=92)\end{array}$ & p-value \\
\hline Screw malposition & $1(1.2 \%)$ & $1(1.1 \%)$ & $0.962, \mathrm{NS}$ \\
Dural tear & $6(6.9 \%)$ & $7(7.6 \%)$ & $0.871, \mathrm{NS}$ \\
Screw loosening & $3(3.5 \%)$ & $4(4.4 \%)$ & $0.767, \mathrm{NS}$ \\
Cage slippage & $3(3.5 \%)$ & $1(1.1 \%)$ & $0.287, \mathrm{NS}$ \\
Implant failure & $1(1.2 \%)$ & $1(1.1 \%)$ & $0.962, \mathrm{NS}$ \\
Nausea/vomiting & $13(15.1 \%)$ & $7(7.6 \%)$ & $0.113, \mathrm{NS}$ \\
Urinary retention & $5(5.8 \%)$ & $19(20.7 \%)$ & $0.004^{*}$ \\
\hline
\end{tabular}

NS: not significant.

${ }^{*} \mathrm{p}<0.05$ 
operation theatre to incision time and bandaging to exit time from the operation theatre. We found a significantly shorter operation theater to incision time and also time from bandaging to the exit in the spinal anesthesia group ( $\mathrm{p}<0.05$ ), which is comparable to study done by Pierce et al. [15]. This highlighted higher efficiency with quicker operation theatre turnover rates and inturn cost effectiveness. The longer duration of general anesthesia in comparison to spinal one was because of the peri-anesthesia events which included pre-anesthestic medication taking time to prepare before induction and intubation as well as post operative anesthesia reversal time which was not there in spinal anesthesia group.

The patients of the spinal anesthesia group required lesser PACU time along with a shorter hospital stay in comparison to the general anesthesia group patients in our study $(\mathrm{p}<0.05)$. Jellish et al. [10] found a longer duration of hospital stay in patients who underwent surgery under general anesthesia. Since this study was performed at lower lumbar levels i.e. below L3, the chance of neurodeficit was very less. Also, author did not experience any event of neurodeficit in his period of study. Although general anesthesia offers the advantage of observation of motor recovery soon after reversal of anesthesia, a sensible motor evaluation is difficult to obtain as patient is in drowsy state. Spinal anesthesia offered excellent control of post operative pain which gave the advantage of early shifting of patient from PACU to ward.

The mean hospital stay in our study was lesser in spinal anesthesia group as compared to general anesthesia $(p<0.05)$ which is comparable to results obtained by Pierce et al. [15]. Early ambulation, early start to oral feeds with less throat irritation, early bowel function return and less neurocognitive changes favoured early discharge from hospital after spinal anesthesia.

We found a significantly higher incidence of urinary retention in spinal anesthesia group ( $20.4 \%$ vs. $5.7 \%$ in general anesthesia), while incidence of nausea/vomiting was comparable ( $p>0.05$ ). In a study done by Jellish et al. [10] the incidence of nausea was significantly higher in spinal anesthesia, while urinary retention incidence was higher in general anesthesia. This finding is contradictory to our findings. McLain et al. [11] found a higher incidence of nausea in general anesthesia group, while reported a significantly lower incidence of urinary retention in spinal anesthesia induced patients.

Papadopoulos et al. [14] also reported a higher incidence of nausea in general anesthesia group. Prolonged analgesia and sensory loss after bupivacaine perhaps could be the reason of higher incidence of urinary retention in our patients after spinal anesthesia as compared to general anesthesia. All those patients who had urinary retention episode had temporary retention. None of the patient had permanent retention. The patients with retention issues were managed by inserting foley's catheter in situ and keeping it for 24 hours which was later removed. Patients were discharged comfortably after urine was passed.

Though it may seem that spinal anesthesia has certain advantages over general anesthesia but this method cannot be recommended for all patients. Loss of spinal anesthesia effect can happen although not reported in any of our patients. Another disadvantage being time constraint in spinal anesthesia group.

Our study too has some flaws. Firstly, intraoperative hemodynamic changes were not recorded; therefore, we could not compare the patients anesthetically. Also, satisfaction rate of the surgeon, anesthesiologist, and the patient was not investigated, although it is one of the important criteria for choosing a certain anesthetic type.

\section{CONCLUSION}

Spinal anesthesia offers efficient operating room functioning with decreasing overall operation theatre time with lower operation theatre entry to incision and bandaging to exit time. Lesser post anesthesia care unit time (PACU) in spinal anesthesia ensures early shift to ward for patients. Lower duration of stay in hospital in spinal anesthesia, lowers down the overall cost for the patient. Hence, spinal anesthesia is very efficient alternative technique to general anesthesia which can be considered for elective lumbar surgeries with a lower late of adverse event especially at lower lumbar levels.

\section{CONFLICT OF INTEREST}

No potential conflict of interest relevant to this article.

\section{REFERENCES}

1. Demirel CB, Kalayci M, Ozkocak I, Altunkaya H, Ozer Y, Acikgoz B. A prospective randomized study comparing perioperative outcome variables after epidural or general anesthesia for lumbar disc surgery. J Neurosurg Anesthesiol 2003;15:185-192.

2. De Rojas JO, Syre P, Welch WC. Regional anesthesia versus general anesthesia for surgery on the lumbar spine: a review of the modern literature. Clin Neurol Neurosurg 2014;119:3943. 
3. Pflug AE, Halter JB. Effect of spinal anesthesia on adrenergic tone and the neuroendocrine responses to surgical stress in humans. Anesthesiology 1981;55:120-126.

4. McLain RF, Bell GR, Kalfas I, Tetzlaff JE, Yoon HJ. Complications associated with lumbar laminectomy: a comparison of spinal versus general anesthesia. Spine (Phila Pa 1976) 2004;29:2542-2547.

5. McLain RF, Tetzlaff JE, Bell GR, Uwe-Lewandrowski K, Yoon HJ, Rana M. Microdiscectomy: spinal anesthesia offers optimal results in general patient population. J Surg Orthop Adv 2007;16:5-11.

6. Rodgers A, Walker N, Schug S, McKee A, Kehlet H, van Zundert A, et al. Reduction of postoperative mortality and morbidity with epidural or spinal anaesthesia: results from overview of randomised trials. BMJ 2000;321:1493.

7. Chen HT, Tsai CH, Chao SC, Kao TH, Chen YJ, Hsu HC, et al. Endoscopic discectomy of L5-S1 disc herniation via an interlaminar approach: prospective controlled study under local and general anesthesia. Surg Neurol Int 2011;2:93.

8. Greenbarg PE, Brown MD, Pallares VS, Tompkins JS, Mann NH. Epidural anesthesia for lumbar spine surgery. J Spinal Disord 1988;1:139-143.

9. Kao FC, Tsai TT, Chen LH, Lai PL, Fu TS, Niu CC, et al. Symptomatic epidural hematoma after lumbar decompression surgery. Eur Spine J 2015;24:348-357.
10. Jellish WS, Thalji Z, Stevenson K, Shea J. A prospective randomized study comparing short- and intermediate-term perioperative outcome variables after spinal or general anesthesia for lumbar disk and laminectomy surgery. Anesth Analg 1996;83:559-564.

11. McLain RF, Kalfas I, Bell GR, Tetzlaff JE, Yoon HJ, Rana M. Comparison of spinal and general anesthesia in lumbar laminectomy surgery: a case-controlled analysis of 400 patients. J Neurosurg Spine 2005;2:17-22.

12. Dashtbani M, Dori MM, Hassani M, Omidi-Kashani F. A survey on the short-term outcome of microlumbar discectomy with general versus spinal anesthesia. Clin Orthop Surg 2019;11:422-426.

13. Sadrolsadat SH, Mahdavi AR, Moharari RS, Khajavi MR, Khashayar P, Najafi A, et al. A prospective randomized trial comparing the technique of spinal and general anesthesia for lumbar disk surgery: a study of 100 cases. Surg Neurol 2009 71:60-65. discussion 65

14. Papadopoulos EC, Girardi FP, Sama A, Pappou IP, Urban MK, Cammisa FP Jr. Lumbar microdiscectomy under epidural anesthesia: a comparison study. Spine J 2006;6:561-564.

15. Pierce JT, Kositratna G, Attiah MA, Kallan MJ, Koenigsberg $\mathrm{R}$, Syre $\mathrm{P}$, et al. Efficiency of spinal anesthesia versus general anesthesia for lumbar spinal surgery: a retrospective analysis of 544 patients. Local Reg Anesth 2017;10:91-98. 